\title{
Effect of a Novel Stretching Technique on Shoulder Range of Motion in Overhead Athletes with Glenohumeral Internal Rotation Deficits: A Randomized Controlled Trial
}

\section{Omar Gharisia}

Loma Linda University School of Allied Health Profession

\section{Everett Lohman}

Loma Linda University School of Allied Health Professions

Noha Daher

Loma Linda University School of Allied Health Profession

\section{Alan Eldridge}

Loma Linda University School of Allied Health Professions

\section{Amjad Shallan}

Faculty of Applied Medical Sciences, The Hashemite University

Hatem Jaber ( $\nabla$ hjaber@llu.edu )

Loma Linda University Adventist Health Sciences Center https://orcid.org/0000-0002-2080-4538

\section{Research article}

Keywords: Glenohumeral Internal Rotation Deficits, Novel Stretch Technique, Overhead Athletes

Posted Date: January 11th, 2021

DOI: https://doi.org/10.21203/rs.2.16116/v4

License: @ (i) This work is licensed under a Creative Commons Attribution 4.0 International License. Read Full License 


\section{Abstract}

Background: The cross-body and the modified sleeper stretch have been used to improve posterior shoulder soft tissue flexibility and to increase glenohumeral joint internal rotation (GHJ IR) in overhead athletes. However, due to the inability to stabilize patient's scapula and control GHJ rotation with the cross-body stretch and the potential for subacromial impingement or symptoms' aggravation with the modified sleeper stretch, a new stretching technique (Passive Glenohumeral Internal Rotation with Clam Shell Bridging) was developed as an alternative to these commonly used stretches that may allow for greater stability of the scapula without reproducing symptoms, as well as providing an inhibition component to the posterior shoulder girdle muscles. Thus, the current study aimed to examine and compare a novel stretching technique to the traditional modified sleeper stretch to determine the effect on glenohumeral IR range of motion (ROM) and self-reported pain in overhead athletes with glenohumeral internal rotation deficits (GIRD).

Methods: Forty-two overhead athletes with GIRD [mean age $25.9 \pm 2.6$ years, 20 males and 22 females] participated in this study. Participants were randomly assigned into either novel stretching group or modified sleeper stretching group. IR ROM was measured with a digital inclinometer before, immediately, and at week 4 post intervention, while pain was measured with Numeric Pain Rating Scale before and at week 4 post intervention.

Results: There was no significant group by time interaction effect for IR ROM ( $p=0.27)$; however, there was a significant change over time $\left(p<0.001, \eta^{2}=0.77\right)$. Both groups demonstrated a significant increase in IR from baseline to immediate and week 4 , and from immediate to week $4(p<0.001)$. There was a significant group by time interaction for pain intensity $\left(p=0.032, \eta^{2}=0.31\right)$. Results showed a significant reduction in pain intensity over time in the novel group $\left(p=0.001, \eta^{2}=0.85\right)$, but not in the traditional group $\left(p=0.150, \eta^{2}=0.27\right)$.

Conclusion: Both stretches appear to be effective at improving IR ROM in overhead athletes with GIRD. However, the novel stretching might be more effective at reducing shoulder pain and thus may be more appropriate for symptomatic patients.

Trial registration: Prospectively registered in February 6, 2017 under Clinical Trial Registry \# NCT03044236

\section{Background}

The shoulder complex is the most commonly injured body part in overhead and throwing athletes, accounting for $30 \%$ of all injuries $(1,2)$. With the growing interest and participation in overhead sports such as baseball, tennis, volleyball, squash, swimming or water polo, the incidence of shoulder pain and/ or injuries continues to increase (3-5). In fact, more than half of the general population will experience shoulder pain at one time or another in their lives (6). Moreover, about $20 \%$ of all disability costs from musculoskeletal abnormalities are related to shoulder problems (7). Compromised shoulder movement due to pain or injury can cause substantial disability and affect a person's ability to carry out daily activities and restricts sport participation.

It has been reported that individuals who frequently participate in overhead throwing sports demonstrate an altered mobility patterns in the throwing shoulder as compared to the non-throwing shoulder (8). These adaptive mobility patterns usually present as excessive external rotation (ER) and decreased internal rotation (IR) of the throwing shoulder (9). This loss of IR range $\left(15^{\circ}-25^{\circ}\right)$ has been defined as Glenohumeral Internal Rotation Deficit 
(GIRD) (10-12) and has been linked to adaptive structural changes in the soft tissue (i.e. capsuloligamentous or muscular tightness) and/or osseous tissue (i.e. humeral retroversion) of the glenohumeral (GH) joint as result of the extreme demands and the excessive overload of repetitive overhead activities (8).

Posterior inferior capsular and rotator-cuff tightness have been suggested as the main contributing factors to the loss of GH IR range of motion (ROM) for most athletes $(8,9)$. The repetitive overhead motions which usually involve excessive ER with a maximally abducted position (cocking phase) and the subsequent phases of acceleration/deceleration and follow-through place a high amount of stress on the static and dynamic stabilizers of the shoulder, including the rotator cuff, joint capsule, and labrum. This repetitive overuse load will ultimately lead to a failure in these structures to absorb arm energy, predisposing them to injury, which generates pain and posterior shoulder tightness $(8,9)$. If left uncorrected, this increased tightness in the posterior structures may cause the humeral head to translate anterosuperiorly during elevation and IR or posterosuperiorly during maximal ER leading to subacromial impingement and/or Type II Superior Labral Anterior to Posterior (SLAP) lesions (13-16). In a study by Burkhart et al (17), pitchers were shown to have a loss of IR in the shoulder with the SLAP lesion compared to the non-injured shoulder. Overhead athletes with shoulder internal impingement were also found to have a reduction in GH IR as a result of posterior shoulder tightness (10). Evidence has suggested that overhead athletes who demonstrate a limitation of $\geq 25^{\circ}$ in shoulder IR are at 4 times higher risk of shoulder or elbow pain and injury while those with a limitation of $\geq 10^{\circ}$ are at approximately 2 times higher risk (13).

Stretching has been proposed as an effective approach for the management of GIRD, restoring shoulder ROM, and reducing the incidence of shoulder injury and muscle soreness $(16,18)$. There are a number of stretching techniques, such as cross-body or horizontal adduction stretches that have been used to improve GHJ ROM (19). Manske et al (20) examined the effect of 4 weeks of cross-body stretching alone versus cross-body stretching and posterior GHJ mobilizations on GHJ IR ROM, and reported similar improvement in GHJ IR for both interventions. However, the inability to stabilize the patient's scapula and control glenohumeral joint rotation while performing the cross-body stretch has made this technique less favorable (21). Nonetheless, a newer stretching technique, the Sleeper Stretch, has been adapted and commonly recommended by clinicians to restore the Glenohumeral Joint (GHJ) IR ROM. Burkhart et al (16) and Launder at al (21) examined the effect of the Sleeper Stretch on shoulder ROM and found that the sleeper stretch group improved significantly in IR ROM compared to the control stretching group. However, a potential for subacromial impingement or aggravation of symptoms with the traditional and the modified sleeper stretch has been documented $(22,23)$.

Although both static stretching techniques (sleeper and cross-body stretches) have been the primary stretches for restoring GHJ IR, the lack of scapular stabilization or the aggravation of symptoms remain a concern for clinicians that prescribe these stretching techniques to patients and clients with GIRD. Given this, we sought to justify a new stretching technique as an alternative to the commonly used stretching techniques that may allow for greater stability of the scapula without aggravating or reproducing symptoms, as well as providing an inhibition component to the posterior shoulder girdle muscles. Thus, the novel technique could provide favorable outcomes. The novel technique is performed while the subject is in a resisted bridging position (Figure 1). Bridging was incorporated into the new technique as an approach to minimize the contact area to mainly the upper back and scapula, thus more pressure is placed on the scapula. That way, scapular movement might be very limited allowing for a proper stretching of the external rotators. 
With the growing evidence regarding the effect of stretching on GIRD, it is important that this novel technique be empirically evaluated, accurately described, and reported to the clinical and scientific communities. Therefore, the current study aimed to examine and compare a novel stretching technique to the traditional modified sleeper stretch to determine the effect on GHJ IR ROM and self-reported pain in overhead athletes with GIRD.

Figure 1. Passive Glenohumeral Internal Rotation with Clam Shell Bridging.

\section{Methods}

\section{Trial design}

This was a parallel-design 2-arm, assessor-blinded randomized controlled clinical trial. Outcomes were assessed at baseline, immediately post intervention, and at week 4 post intervention. The study's protocol was in compliance with the CONSORT guidelines.

\section{Participants}

A sample of 42 physically active adult athletes (20 males, 22 females) voluntarily participated in this study. Participants were recruited by flyers, emails, referrals, and word of mouth from a college campus and a community in Sothern California. All participants read and signed a written informed consent approved by the Institutional Review Board of Loma Linda University prior to participation. Eligible participants met the following inclusion criteria: 1) between 18 and 45 years of age; 2) performed overhead sports activities, such as volleyball, tennis, water polo, squash, baseball, swimming, or lifting as a major activity of interest; 3) participated in local and regional sport competitions 4 ) displayed a $10^{\circ}$ or greater of between-shoulder difference in IR ROM (dominant versus non-dominant); and 5) with or without pain during shoulder activities. These criteria were selected based on a recent systematic review, which suggested that a lower threshold ROM deficit, based on a weighted mean GIRD of $13.8^{\circ}$ among all injured athletes, should be utilized if the goal is to improve ROM and prevent injuries in these athletes (24). Subjects were excluded if they were: 1 ) still recovering from previous surgery of the shoulder and elbow complex in the past 3 months; 2) currently receiving medical intervention for the shoulder; or 3) have any critical medical condition. All experimental procedures were conducted in the orthopedic laboratory at Loma Linda University, Department of Physical Therapy from April 2017 to November 2018.

\section{Instrumentation}

A digital inclinometer (MicroFET3, Draper, UT) was used to measure GH IR and ER ROMs. This instrument has been shown to have a high intra-rater and inter-rater reliability [Intraclass correlation coefficient $($ ICC) $=0.87$ and ICC $=0.93$, respectively] (25). Subsequently, a two-pound pressure, via an electronic push/pull dynamometer (Baseline Electronic Push/Pull Dynamometer - MicroFET3, Draper, UT), was applied by the same examiner at the end of available passive range of motion prior to taking a ROM measurement in order to standardize the shoulder internal and external rotation values. Pain was measured using the Numeric Pain Rating Scale (NPRS). NPRS has been shown to be valid, reliable, and appropriate for use in clinical practice (26). 


\section{Procedures and intervention}

Forty subjects who fulfilled the inclusion criteria were recruited. After stratification, participants were randomly assigned to one of the 2 intervention groups. Group A comprised the modified sleeper stretch (MSS) group ( $n=$ 22) (Figure 2) while Group B comprised the novel stretch (NS) group $(n=20)$ (Figure 1). Group assignment was conducted by an independent person using a random number generator and concealed in sealed envelopes from all personnel involved in screening before randomization. Post randomization, objective measures' assessors were blinded to group assignment. Participants were taught their assigned home-based stretching program by the investigator who explained the stretching technique to each participant. All participants were asked to demonstrate the stretch to ensure mastery. Additionally, to ensure and facilitate compliance, participants were provided a written sheet with detailed instructions and demonstrative pictures of the stretch. A log sheet was provided to monitor the number of stretches per week. Participants were also contacted weekly through phone calls and text messages as a reminder to minimize lack of compliance. All participants performed 4-weeks of stretching protocol, three times a week, with three repetitions, holding each stretch for 30 seconds, with 30 seconds rest between repetitions (27).

Figure 2. Modified Sleeper Stretch: Passive Glenohumeral Joint Internal Rotation.

Participants in Group A were instructed to perform the MSS in a side-lying position, on the side to be stretched, with the elbow flexed to $90^{\circ}$, shoulder elevated to $90^{\circ}$, and the trunk slightly rolled posteriorly $20^{\circ}$ to $30^{\circ}$ to place the shoulder in the scapular plane as passive IR is performed (Figure 2). Participants were instructed to allow the stretched shoulder to naturally fall into IR to the end ROM where resistance could be felt. Participants were then instructed to use the other hand to push the stretched shoulder into further IR to the point of mild stretch discomfort by applying pressure at the area of the wrist joint.

Participants in Group B were instructed to perform the NS in a supine position. Participants placed a resistance band around their knees and were instructed to open their knees. Participants were asked to bridge as high as possible, with the elbow flexed to $90^{\circ}$ and shoulder abducted to $90^{\circ}$ (Figure 1). The bridging maneuver shifts the body weight superiorly pinning the medial border of scapula against the thorax without directly compressing or restricting posterior shoulder structures. It is proposed that this position will allow greater freedom of motion with less discomfort. While maintaining this position, participants were asked to contract or "squeeze" their gluteal muscles and perform the stretching by actively internally rotating their shoulder to the end of ROM. Participants used the other hand to push to the point of mild stretch discomfort and simultaneously maintain contraction while progressing the stretch.

Shoulder IR was measured by positioning the participant in a side lying position, with the elbow flexed to $90^{\circ}$ and shoulder abducted to $90^{\circ}$. Participants were instructed to allow the shoulder to naturally fall into internal rotation to the end ROM where resistance was felt. At this point, an electronic push/pull dynamometer was applied to the distal forearm at the end range of motion. Before recording the ROM measurement, the physical therapist applied consistent pressure (2 pounds) in an attempt to standardize and quantify the passive force to ensure reliable and valid ROM measurements. The digital inclinometer was then aligned along the ulna of the 
stretched arm and three trials of the internal rotation ROM measurements were taken, averaged and used for analysis.

For subjects experiencing shoulder pain, the intensity of pain was measured using the NPRS. An experienced physical therapist provided a detailed explanation regarding the NPRS prior to the administration of the NPRS questionnaire to ensure better understanding. All participants had to complete the NPRS questionnaire at baseline and post 4-weeks.

\section{Statistical Analyses}

A sample size of 42 participants was estimated using a moderate effect size for the group $\mathrm{x}$ time interaction (partial $\left.\eta^{2}=0.06\right)(28)$, level of significance $(a=0.05)$, power of 0.80 , and a $35 \%$ dropout.

Data was summarized using mean and standard deviation for quantitative variables and counts (\%) for qualitative variables. The normality of continuous variables was examined using Shapiro Wilk's test and Q-Q normality plots. The distribution of the participants' characteristics by study group was evaluated using chisquare for qualitative variables, independent t-test for quantitative variables and Mann-Whitney test for ordinal variables. A 2-group $\times$ 3-time points (baseline, week 1, and week 4) mixed factorial analysis of variance (ANOVA) was used to examine changes in mean GH IR ROM and pain intensity (2x2, baseline vs. week 4) by study group over time.

The primary analysis included a comparison between groups using the group $x$ time interaction effect. If the interaction was statistically significant, change from baseline was compared between groups at each follow-up time point using an independent t-test. If the results were not statistically significant, the between-groups comparison was considered not statistically significant at any time point. However, Bonferroni post hoc test was conducted on the combined groups only if the main effect of time was significant in the mixed factorial ANOVA.

The secondary analysis included testing of change from baseline at each time point within-groups using oneway repeated measures ANOVA. If the results of the test were statistically significant, post hoc comparisons using Bonferroni test was conducted on each study group separately. Partial Eta Squared (n2) was reported for significant differences in ANOVA and categorized as [0.01 small, 0.06 medium, and $\geq 0.13$ large], while Cohen's $\mathrm{d}$ effect size was used for independent t-test and categorized as [0.2 small, 0.5 medium, and $\geq 0.8$ large] (29). The level of significance was set at $p \leq 0.05$. Statistical analysis was performed using IBM SPSS Software version 25 for Windows (Chicago, IL, USA).

\section{Results}

Forty-two participants with mean age $25.9 \pm 2.6$ years old and body mass index (BMI) of $19.0 \pm 4.2 \mathrm{~kg} / \mathrm{m}^{2} \mathrm{were}$ recruited. Two participants dropped out from the MSS group following week 1 of intervention with unknown reasons, and 40 completed the study (Figure 3). Fifty-five percent of the participants were females $(n=22)$. The mean IR deficit was $15.6 \pm 5.5$. Nonetheless, the analysis was performed on all available data from all randomized participants according to the intention-to-treat principle (20 NS and 22 MSS). The compliance rate 
of home-stretching program was $100 \%$ for both groups. In addition, none of the participants reported receiving any outside care during the study period. The distribution of the continuous variables was approximately normal. There were no significant differences in demographic and baseline outcome measures between the two study groups $(p>0.05)$, Table 1 . None of the participants reported any harm or adverse effects following interventions.

Figure 3. Flow diagram of participants' recruitment and retention.

Table 1. Mean (SD) of General Characteristics and Baseline Outcomes $(\mathrm{N}=40)$.

\begin{tabular}{|l|c|c|}
\hline & Novel Stretching $\left(\mathrm{n}_{1}=20\right)$ & Traditional $\left(\mathrm{n}_{2}=22\right)$ \\
\hline Female; n (\%) & $11(55)$ & $11(55)$ \\
\hline Age (years) & $26.0(2.5)$ & $25.9(2.6)$ \\
\hline BMI (kg/m $\left.{ }^{2}\right)$ & $27.3(4.5)$ & $25.1(3.5)$ \\
\hline GIRD (degree) & $15.8(5.1)$ & $15.4(5.3)$ \\
\hline Compliance Rate (\%) & $100(0)$ & $100(0)$ \\
\hline Baseline Pain & $4.43(1.4)$ & $49.6(15.7)$ \\
\hline GHIR_ROM (degree) & $49.2(12.7)$ & \\
\hline
\end{tabular}

Abbreviations: SD, Standard Deviation; GHIR_ROM, Glenohumeral Internal Rotation Range of Motion Results of the mixed factorial ANOVA are displayed in Table 2. There was no significant group by time interaction effect for GH IR ROM ( $p=0.27)$; however, there was a significant change over time $\left(p<0.001, \eta^{2}=0.77\right)$ for the combined groups. Bonferroni post hoc comparison revealed that IR ROM significantly increased from baseline to immediate (49.4 \pm 14.1 vs. $63.1 \pm 13.2$, $p<0.001)$ and week $4(49.4 \pm 14.1$ vs. $70.0 \pm 12.7, p<0.001)$, and from immediate to week 4 (63.1 \pm 13.2 vs. $70 \pm 12.7, p<0.001)$. Similar results were obtained after controlling for gender [there was no significant interaction $(p=0.25)$, but there was a significant change over time $(p<0.001)$ ].

Table 2. Mean (SD) of Glenohumeral Internal ROM (N) by Study Group over Time (N=42). 


\begin{tabular}{|c|c|c|c|c|c|c|c|c|}
\hline & \multicolumn{3}{|c|}{ Novel Stretching $\left(\mathrm{n}_{1}=20\right)$} & \multicolumn{3}{|c|}{ Traditional Stretching $\left(\mathrm{n}_{2}=22\right)$} & \multirow{2}{*}{$\begin{array}{c}\text { p-value over } \\
\text { time }^{*}\left(\eta^{2}\right)\end{array}$} & \multirow{2}{*}{$\begin{array}{l}\text { p-value (group x } \\
\text { time) }^{*}\left(\eta^{2}\right)\end{array}$} \\
\hline & Baseline & Immediate & $\begin{array}{c}4 \text { weeks } \\
\text { later }\end{array}$ & Baseline & Immediate & $\begin{array}{c}4 \\
\text { weeks } \\
\text { later }\end{array}$ & & \\
\hline GHIR_ROM ${ }^{\mathrm{a}}$ & $\begin{array}{c}49.2 \\
(12.7)\end{array}$ & $\begin{array}{c}63.7 \\
(10.3)\end{array}$ & $\begin{array}{c}72.0 \\
(10.6)\end{array}$ & $\begin{array}{c}49.6 \\
(15.7)\end{array}$ & $\begin{array}{c}62.6 \\
(15.8)\end{array}$ & $\begin{array}{c}68.1 \\
(14.5)\end{array}$ & $\begin{array}{c}<0.001 \\
(0.77)\end{array}$ & $0.27(0.03)$ \\
\hline
\end{tabular}

bbreviations: SD, Standard Deviation; GHIR_ROM, Glenohumeral Internal Rotation Range of Motion; $\eta^{2}$, Partial Eta quared

Mixed Factorial ANOVA, level of significance was set at $\mathrm{p} \leq 0.05$

Significant difference between baseline \& immediate, baseline \& week 4, and immediate \& week $4(\mathrm{p}<0.001)$

For pain intensity, none of those who were asymptomatic reported any pain 4 weeks post-intervention. However, for those who were symptomatic at baseline ( 7 novel, 8 traditional), there was a significant group by time interaction $\left(p=0.032, \eta^{2}=0.31\right)$, Table 3 . Results of the independent t-test showed a significant difference between the two groups at week 4 post intervention $(p=0.032, d=1.26)$, Table 4 . Results of the one-way repeated measures ANOVA for the within-group analysis showed a significant reduction in pain intensity over time in the novel group $\left(p=0.001, \eta^{2}=0.85\right)$. However, there was no significant change in pain intensity over time for the traditional group $\left(p=0.150, \eta^{2}=0.27\right)$, Table 3 . It is note mentioning that though pain analysis was based on a smaller sample size $(\mathrm{N}=15)$, post hoc power analysis using the effect size of 0.31 that was found in pain intensity interaction effect, revealed that the observed power was 0.99 .

\section{Table 3. Mean (SD) of Pain Intensity by Study Group over Time ( $=15)$.}

\begin{tabular}{|c|c|c|c|c|c|c|}
\hline & \multicolumn{2}{|c|}{$\begin{array}{l}\text { Novel Stretching } \\
\left(\mathrm{n}_{1}=7\right)^{\mathrm{a}}\end{array}$} & \multicolumn{2}{|c|}{$\begin{array}{l}\text { Traditional Stretching } \\
\qquad\left(\mathrm{n}_{2}=15\right)\end{array}$} & \multirow[t]{2}{*}{$\begin{array}{l}\text { p-value over } \\
\text { time }^{*}\left(\eta^{2}\right)\end{array}$} & \multirow[t]{2}{*}{$\begin{array}{c}\text { p-value (group } x \text { time) }{ }^{*} \\
\left(\eta^{2}\right)\end{array}$} \\
\hline & Baseline & $\begin{array}{c}4 \text { weeks } \\
\text { later }\end{array}$ & Baseline & 4 weeks later & & \\
\hline $\begin{array}{c}\text { Pain } \\
\text { Intensity }\end{array}$ & $\begin{array}{l}4.43 \\
(1.4)\end{array}$ & $0.71(0.49)$ & $3.00(1.31)$ & $1.75(1.5)$ & $<0.001(0.65)$ & $0.032(0.31)$ \\
\hline
\end{tabular}

bbreviations: SD, Standard Deviation; $\eta^{2}$, Partial Eta Squared

Mixed Factorial ANOVA, level of significance was set at $\mathrm{p} \leq 0.05$

jignificant difference between baseline \& week $4(\mathrm{p}=0.001)$

able 4. Mean (SD) Change of Pain Intensity from Baseline by Study Group ( $N=15)$. 


\begin{tabular}{|c|c|c|c|c|c|c|}
\hline & $\begin{array}{c}\text { Time Change from } \\
\text { Baseline }\end{array}$ & $\begin{array}{c}\text { Novel } \\
\text { Stretching } \\
\left(\mathrm{n}_{1}=7\right)\end{array}$ & $\begin{array}{c}\text { Traditional } \\
\text { Stretching }\left(\mathrm{n}_{2}=8\right)\end{array}$ & $\begin{array}{c}\text { Mean Difference } \\
(95 \% \mathrm{CI})\end{array}$ & $\begin{array}{c}\text { Ef- } \\
(\text { Cohen's d) }\end{array}$ \\
\hline $\begin{array}{c}\text { Pain } \\
\text { Intensity }\end{array}$ & Week 4 & $3.72(1.7)$ & $1.25(2.2)$ & $2.47(0.25,4.7)$ & 0.032 & 1.26 \\
& & & & \\
\hline
\end{tabular}

Abbreviation: SD, Standard Deviation; CI, Confidence Interval

*Independent t-Test, level of significance was set at $\mathrm{p} \leq 0.05$

\section{Discussion}

\section{Glenohumeral Internal Rotation Range of Motion}

The results of the current study suggest that in overhead athletes with GIRD, the NS technique (Passive Glenohumeral Internal Rotation with Clam Shell Bridging) is no more effective than the traditional MSS at improving GH IR ROM. Both groups demonstrated significant increases in IR from baseline to immediate and week 4, and from immediate to week 4. It can be inferred that the increases noted in both groups reflect a real increase in IR taking into consideration the minimal detectable change $\left(\mathrm{MDC}_{95}\right)$ values we obtained. The MDC represents the smallest change in measurement over time that reflects a true threshold change rather than simple measurement error (30). The $\mathrm{MDC}_{95}$ values reported in this study indicated that a change greater than or equal to $9.3^{\circ}$ was required to be $95 \%$ certain that the change was not due to subject/intertrial variability or measurement error. The changes from baseline to immediate were NS $14.5^{\circ}$, MSS $13^{\circ}$ and from baseline to week 4 were NS $22.8^{\circ}$, MSS $18.5^{\circ}$, which exceeded the obtained MDC value. Similarly, Kolber et al $(31,32)$ have suggested that a $\mathrm{MDC}_{90}$ value of $8^{\circ}-9^{\circ}$ should be considered when interpreting change values over subsequent treatment sessions. However, given that we did not calculate the minimal clinically important difference (MCID), which represents the smallest amount of change that is clinically meaningful as perceived by the patient (33), and that there is no reported reference (threshold) value for MCID in literature, we are uncertain as to what extent the obtained change would be considered clinically meaningful.

Though we did not re-examine whether or not the side-to-side differences in IR still existed following the stretching techniques as we only measured the improvement in the treated dominant side over time, the proportion of those who showed an improvement in IR that reached or exceeded the mean IR deficit of $15.6^{\circ}$ was high, $80 \%$ (90\% in novel vs. $75 \%$ in traditional). This indicates that such differences would have been diminished/disappeared post intervention.

Comparing these results with other research findings, however, is difficult because of the identical lack of studies that used/compared the same stretching procedures. However, the clinical merits of these stretching techniques could be established by comparing the outcomes of this study with previous relevant studies. Comparative studies have been carried out to determine effects of various stretching techniques on shoulder ROM deficit. 
Laudner et al (21) reported an immediate increase in IR ROM following passive sleeper stretches performed 3 times for 30 seconds. Bailey et al (23) and Mine K (34) reported a significantly immediate increase in IR using the cross-body and the sleeper stretches, performed 2 to 5 times for 20 s to 1 minute. In addition, McClure et al (35) reported significant improvement in IR following 4 weeks of cross-body stretch and sleeper stretch, with greater increase noted for the cross-body stretch. The findings of the present study are in agreement with the above research studies that showed stretching is an effective method to improve ROM deficits $(21,27,35)$. The observed gains in IR ROM between baseline and immediate (NS 14.5 , MSS $13^{\circ}$ ) and baseline and week 4 (NS $22.8^{\circ}, \mathrm{MSS} 18.5^{\circ}$ ) in our study are comparable to those observed in these studies (range $7^{\circ}$ to $15^{\circ}$ ) $(34)$.

\section{Pain Intensity}

Only 15 participants (7 novel, 8 traditional) had pain symptoms at baseline. However, a significant reduction (4.43 vs. $0.71,37 \%$ drop form baseline) in pain intensity was observed only in the NS group, while the traditional MSS group remained essentially stable. A 30-35\% reduction in pain from baseline or a decrease of 1.5 to 1.6 on a 10-point NPRS can be considered significant and was rated as MCID (believed to be meaningful by the patients) (36-38). Previous research showed symptoms (pain) relief after stretching program in athletes with GIRD and impingement-related shoulder pain (39). In contrast, McClure et al (35) reported frequent pain with the sleeper stretch as opposed to the cross-body stretch. However, in order to minimize symptoms of pain aggravation and discomfort associated with the sleeper stretch, we sought to use the MSS (40). This technique was shown to be as effective as the cross-body stretch at improving IR in persons with GIRD (23). Our symptomatic participants in the MSS group did not complain or report any aggravation of symptoms during stretching but pain levels remained essentially stable at week 4, which might further support using it over the traditional sleeper stretch. Nonetheless, it is worth mentioning that in Mine K study (23), 7 participants experienced pain during the MSS and therefore were excluded from the study despite the fact that they were asymptomatic before stretching. In contrast, none of the asymptomatic participants in our study reported any pain during or at the end of the protocol. This discrepancy leaves a gap for future research to further investigate and reach a clear conclusion that could help clinicians to make wise decisions that benefit their patients with less adverse effects.

However, given that there was no significant difference between the effects of the MSS and the NS for IR ROM and that the latter was more effective than the MSS at reducing pain, the NS might have better clinical utility, particularly for patients with shoulder pain. In addition, despite previous research studies having shown that the cross-body stretch is more effective than the traditional sleeper stretch (35) and less provocative than both traditional or MSS $(23,35)$, we chose not to compare the novel technique with the cross-body stretching due to the reported difficulty or inability to stabilize the patients' scapula (21), which may, at some point, bias the results, especially since our protocol was home-based. However, we still recommend that future studies consider comparing both techniques.

Bridging was incorporated into the new technique as an approach to minimize the contact area, thus more pressure is placed on the scapula without posterior shoulder contact, which might limit scapular movement allowing for a proper stretching of the external rotators. Future studies using pressure sensor analysis system, 
however, are recommended to validate this hypothetical assumption. Furthermore, the novel stretch is performed in a supine (bridging) position and thus mechanical stress or discomfort to the subacromial tissues may not likely occur as opposed to that with the traditional or modified sleeper stretch $(22,23)$.

\section{Limitations}

A limitation of this study was the selection of lower threshold GIRD values. However, there is no consensus in literature with respect to the current cut-off values (range, $\left.18^{\circ}-25^{\circ}\right)$ for $\operatorname{GIRD}(12,16,41)$ depending on the study design and population. Therefore, in view of maximal protection of the athlete, it is advised that a lower threshold of less than $18^{\circ}$ be used $(24,41)$. In fact, lower threshold GIRD of $>10^{\circ}$ was used by other studies in an attempt to include a wide range of athletes who could potentially benefit from the stretching program $(23,42)$. In addition to the above reasons, we selected a lower value because we were interested in demonstrating the possible effectiveness of the novel stretching in general before its application to a more vulnerable population who might be at higher risk of injury. Another limitation is that the inclusion of heterogeneous sample of athletes with different sport activities might have potentially biased our findings. Furthermore, the lack of control group leaves a room for speculation that the prestretching ROM assessment, performed by passively moving the athlete's shoulder to an end-range, may have resulted in tissue stretching and thereby affected the poststretching (immediate) ROM measurement. However, in a similar study by Laudner et al (21), the ROM in the true control group (received no intervention) did not differ between the prestretching and poststretching measurements which might indicate that the pre-assessment has no evident or measurable effect on ROM. Additionally, the duration of shoulder pain, for subjects reporting pain at baseline, was not collected at the initial examination to determine the chronicity of pain, and thus the study might have included a mix of subacute and chronic population, and the outcome could be different for these populations in the first place. However, all participants had a duration of more than two weeks of shoulder pain. Finally, the lack of follow up to determine which technique would have a lasting effect on the outcomes, and the smaller sample size for the pain variable, which may limit the statistical power. Though the observed post hoc power for pain intensity was shown to be high, we still recommend future studies with a larger sample size and a follow-up to further enhance the generalizability of the study's findings. Future research should also investigate if adding this active stretch to an overhead athlete's stretching regimen would have any negative impact on the athlete's upper extremity strength.

\section{Conclusions}

Both the NS and the MSS appeared to be similarity effective at improving IR ROM in overhead athletes with GIRD. However, the NS may be more effective than the MSS at reducing shoulder pain and thus might be more appropriate for symptomatic patients. Our findings indicate that 3 sets of 30 second stretching bouts might be sufficient in improving IR ROM. Future research should include prospective studies that further assess and validate the effectiveness of the novel stretching as a rehabilitative intervention in a variety of patient population and as a preventive tool for asymptomatic overhead athletes.

\section{Clinical Implications}

The findings of the present study help to guide clinicians in the selection of the best available stretching option(s) for improving shoulder IR deficit. The NS and the MSS techniques are effective at improving IR ROM in 
overhead athletes with GIRD. However, given that the NS is more effective at reducing shoulder pain, the NS might have better clinical utility, particularly for patients with shoulder pain. When shoulder motion is impaired, its restoration should be a key component of rehabilitation to prevent any potential future injuries. The validation of this novel inhibitory self-stretching technique gives the clinician the option of now giving their clients a self-assisted stretching technique as a part of their home or gym stretching program. This also gives trainers the option of adding this technique to their overhead athletes' pre-season or mid-season stretching regimen with greater confidence that stretching will not cause or exacerbate symptoms.

\section{Abbreviations}

External Rotation (ER)

Internal Rotation (IR)

Glenohumeral Internal Rotation Deficit (GIRD)

Glenohumeral (GH)

Range of Motion (ROM)

Glenohumeral Joint (GHJ)

Numeric Pain Rating Scale (NPRS)

Novel Stretch (NS)

Modified Sleeper Stretch (MSS)

Body Mass Index (BMI)

Minimal Detectable Change $\left(\mathrm{MDC}_{95}\right)$

Minimal Clinically Important Difference (MCID)

Superior Labral Anterior Posterior (SLAP)

\section{Declarations}

\section{Ethical Approval}

Ethical approval was obtained from the Institutional Review Board at LLUH \# 5170032. All subjects read and signed a written informed consent prior to participation in the study.

\section{Consent for publication}

Clinical data are entirely unidentifiable within the manuscript. Thus, obtaining consent for publication was deemed unnecessary. However, a written informed consent for publication of clinical images was obtained from the patient. A copy of the consent form is available for review by the editorial of this journal. 


\section{Availability of data and materials}

The data that support the findings of this study are presented within the manuscript. However, all relevant raw data are available from LLUH but restrictions apply to the availability of these data, which were used under license for the current study, and so are not publically available. Data are however available from the authors upon reasonable request and with permission of LLUH.

\section{Competing interests}

The authors declare that they have no competing interests.

\section{Funding}

This research did not receive any specific grant from funding agencies in the public, commercial, or not-for-profit sectors.

\section{Authors' contributions}

OG contributed to the conception, participated in the design, managed the data collection, assisted in data interpretation, and contributed in writing and revising the manuscript.

EL contributed to the conception, participated in the design, managed the data collection, assisted in data interpretation, and substantially revised the manuscript.

ND participated in the design, managed the data collection, performed the statistical analysis, and substantially revised the manuscript.

AR participated in the design and assisted in revising the manuscript.

AS assisted with the data collection.

$\mathrm{HJ}$ contributed to the conception, participated in the design, managed the data collection, assisted in the statistical analysis, interpreted the data, and was a major contributor in writing and revising the manuscript.

All authors read and approved the final manuscript.

\section{Acknowledgments}

We would like to thank all participants who volunteered their time to advance scientific inquiry.

\section{References}

1. Laudner K, Sipes R. The incidence of shoulder injury among collegiate overhead athletes. Journal of Intercollegiate Sport. 2009;2(2):260-8.

2. Lin DJ, Wong TT, Kazam JK. Shoulder injuries in the overhead-throwing athlete: epidemiology, mechanisms of injury, and imaging findings. Radiology. 2018;286(2):370-87. 
3. Leonard J, Hutchinson MR. Shoulder injuries in skeletally immature throwers: review and current thoughts. British journal of sports medicine. 2010;44(5):306-10.

4. Bonza JE, Fields SK, Yard EE, Dawn Comstock R. Shoulder injuries among United States high school athletes during the 2005-2006 and 2006-2007 school years. Journal of athletic training. 2009;44(1):76-83.

5. Shankar PR, Fields SK, Collins CL, Dick RW, Comstock RD. Epidemiology of high school and collegiate football injuries in the United States, 2005-2006. The American journal of sports medicine. 2007;35(8):1295303.

6. Luime J, Koes B, Hendriksen I, Burdorf A, Verhagen A, Miedema H, et al. Prevalence and incidence of shoulder pain in the general population; a systematic review. Scandinavian journal of rheumatology. 2004;33(2):73-81.

7. Nygren A, Berglund A, Von Koch M. Neck-and-shoulder pain, an increasing problem. Strategies for using insurance material to follow trends. Scandinavian journal of rehabilitation medicine Supplement. 1995;32:107.

8. Borsa PA, Laudner KG, Sauers EL. Mobility and stability adaptations in the shoulder of the overhead athlete. Sports medicine. 2008;38(1):17-36.

9. Lintner D, Mayol M, Uzodinma O, Jones R, Labossiere DJTAjosm. Glenohumeral internal rotation deficits in professional pitchers enrolled in an internal rotation stretching program. 2007;35(4):617-21.

10. Myers JB, Laudner KG, Pasquale MR, Bradley JP, Lephart SM. Glenohumeral range of motion deficits and posterior shoulder tightness in throwers with pathologic internal impingement. The American journal of sports medicine. 2006;34(3):385-91.

11. Lintner D, Mayol M, Uzodinma O, Jones R, Labossiere D. Glenohumeral internal rotation deficits in professional pitchers enrolled in an internal rotation stretching program. The American journal of sports medicine. 2007;35(4):617-21.

12. Shanley E, Michener LA, Ellenbecker TS, Rauh MJ. Shoulder range of motion, pitch count, and injuries among interscholastic female softball pitchers: a descriptive study. International journal of sports physical therapy. 2012;7(5):548.

13. Shanley E, Rauh MJ, Michener LA, Ellenbecker TS, Garrison JC, Thigpen CA. Shoulder range of motion measures as risk factors for shoulder and elbow injuries in high school softball and baseball players. The American journal of sports medicine. 2011;39(9):1997-2006.

14. Dutton M. Orthopaedics for the physical therapist assistant: Jones \& Bartlett Publishers; 2011.

15. Grossman MG, Tibone JE, McGarry MH, Schneider DJ, Veneziani S, Lee TQ. A cadaveric model of the throwing shoulder: a possible etiology of superior labrum anterior-to-posterior lesions. JBJS. 2005;87(4):824-31.

16. Burkhart SS, Morgan CD, Kibler WB. The disabled throwing shoulder: spectrum of pathology Part I: pathoanatomy and biomechanics. Arthroscopy: The Journal of Arthroscopic Related Surgery. 2003;19(4):404-20.

17. Burkhart SS, Morgan CD, Kibler WB. Shoulder injuries in overhead athletes: the "dead arm" revisited. Clinics in sports medicine. 2000;19(1):125-58.

18. Kibler W, Chandler T. Range of motion in junior tennis players participating in an injury risk modification program. Journal of science medicine in sport. 2003;6(1):51-62. 
19. Meister K. Injuries to the shoulder in the throwing athlete: part two: evaluation/treatment. The American journal of sports medicine. 2000;28(4):587-601.

20. Manske RC, Meschke M, Porter A, Smith B, Reiman M. A randomized controlled single-blinded comparison of stretching versus stretching and joint mobilization for posterior shoulder tightness measured by internal rotation motion loss. Sports Health. 2010;2(2):94-100.

21. Laudner KG, Sipes RC, Wilson JT. The acute effects of sleeper stretches on shoulder range of motion. Journal of athletic training. 2008;43(4):359-63.

22. Wilk KE, Hooks TR, Macrina LC. The modified sleeper stretch and modified cross-body stretch to increase shoulder internal rotation range of motion in the overhead throwing athlete. journal of orthopaedic sports physical therapy. 2013;43(12):891-4.

23. Mine K. Immediate effects of two types of stretching techniques on glenohumeral internal rotation deficit and posterior shoulder tightness; a crossover randomised controlled trial. Phys Ther Sports Med. 2017;1:3.

24. Johnson JE, Fullmer JA, Nielsen CM, Johnson JK, Moorman III CT. Glenohumeral internal rotation deficit and injuries: A systematic review and meta-analysis. Orthopaedic journal of sports medicine.

2018;6(5):2325967118773322.

25. Kolber MJ, Corrao M. Shoulder joint and muscle characteristics among healthy female recreational weight training participants. The Journal of Strength Conditioning Research. 2011;25(1):231-41.

26. Williamson A, Hoggart B. Pain: a review of three commonly used pain rating scales. Journal of clinical nursing. 2005;14(7):798-804.

27. Oyama S, Goerger CP, Goerger BM, Lephart SM, Myers JB. Effects of non-assisted posterior shoulder stretches on shoulder range of motion among collegiate baseball pitchers. Athletic Training Sports Health Care. 2010;2(4):163-70.

28. Lakens D. Calculating and reporting effect sizes to facilitate cumulative science: a practical primer for ttests and ANOVAs. Frontiers in Psychology. 2013;(4):863.

29. Cohen J. Statistical power analysis for the behavioral sciences. Academic press; 2013.

30. Schmitt JS, Di Fabio RP. Reliable change and minimum important difference (MID) proportions facilitated group responsiveness comparisons using individual threshold criteria. Journal of clinical epidemiology. 2004;57(10):1008-18.

31. Kolber MJ, Hanney WJ. The reliability, minimal detectable change and construct validity of a clinical measurement for identifying posterior shoulder tightness. North American journal of sports physical therapy: NAJSPT. 2010;5(4):208.

32. Kolber MJ, Vega Jr F, Widmayer K, Cheng M-SS. The reliability and minimal detectable change of shoulder mobility measurements using a digital inclinometer. hysiotherapy Theory Practice. 2011;27(2):176-84.

33. Bernstein JA, Mauger DT. The minimally clinically important difference (MCID): what difference does it make? The Journal of Allergy Clinical Immunology: : In Practice. 2016;4(4):689-90.

34. Bailey LB, Thigpen CA, Hawkins RJ, Beattie PF, Shanley E. Effectiveness of manual therapy and stretching for baseball players with shoulder range of motion deficits. Sports health. 2017;9(3):230-7.

35. McClure P, Balaicuis J, Heiland D, Broersma ME, Thorndike CK, Wood A. A randomized controlled comparison of stretching procedures for posterior shoulder tightness. ournal of orthopaedic sports physical 
therapy. 2007;37(3):108-14.

36. Lee JS, Hobden E, Stiell IG, Wells GA. Clinically important change in the visual analog scale after adequate pain control. Academic Emergency Medicine. 2003;10(10):1128-30.

37. Hao Q, Devji T, Zeraatkar D, Wang Y, Qasim A, Siemieniuk RA, et al. Minimal important differences for improvement in shoulder condition patient-reported outcomes: a systematic review to inform a BMJ Rapid Recommendation. BMJ. 2019;9(2):e028777.

38. Simovitch R, Flurin P-H, Wright T, Zuckerman JD, Roche CP. Quantifying success after total shoulder arthroplasty: the minimal clinically important difference. Journal of shoulder elbow surgery. 2018;27(2):298305.

39. Cools AM, Johansson FR, Cagnie B, Cambier DC, Witvrouw EE. Stretching the posterior shoulder structures in subjects with internal rotation deficit: comparison of two stretching techniques. Shoulder Elbow. 2012;4(1):56-63.

40. Wilk KE, Macrina LC, Fleisig GS, Porterfield R, Simpson CD, Harker P, et al. Correlation of glenohumeral internal rotation deficit and total rotational motion to shoulder injuries in professional baseball pitchers. 2011;39(2):329-35.

41. Wilk KE, Macrina LC, Fleisig GS, Porterfield R, Simpson CD, Harker P, et al. Correlation of glenohumeral internal rotation deficit and total rotational motion to shoulder injuries in professional baseball pitchers. The American Journal of Sports Medicine. 2011;39(2):329-35.

42. Salamh PA, Kolber MJ, Hanney WJ. Effect of scapular stabilization during horizontal adduction stretching on passive internal rotation and posterior shoulder tightness in young women volleyball athletes: a randomized controlled trial. Archives of physical medicine rehabilitation. 2015;96(2):349-56.

\section{Figures}

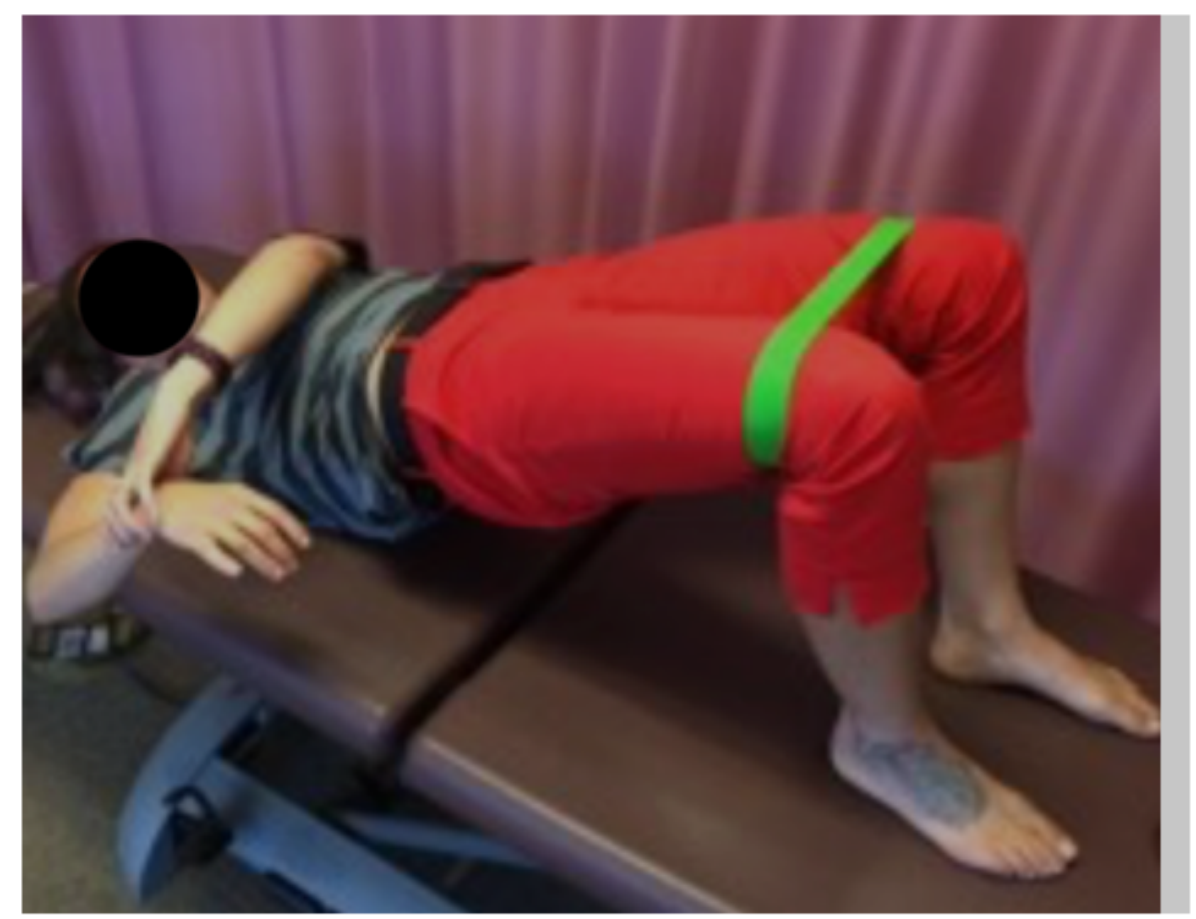




\section{Figure 1}

Passive Glenohumeral Internal Rotation with Clam Shell Bridging.

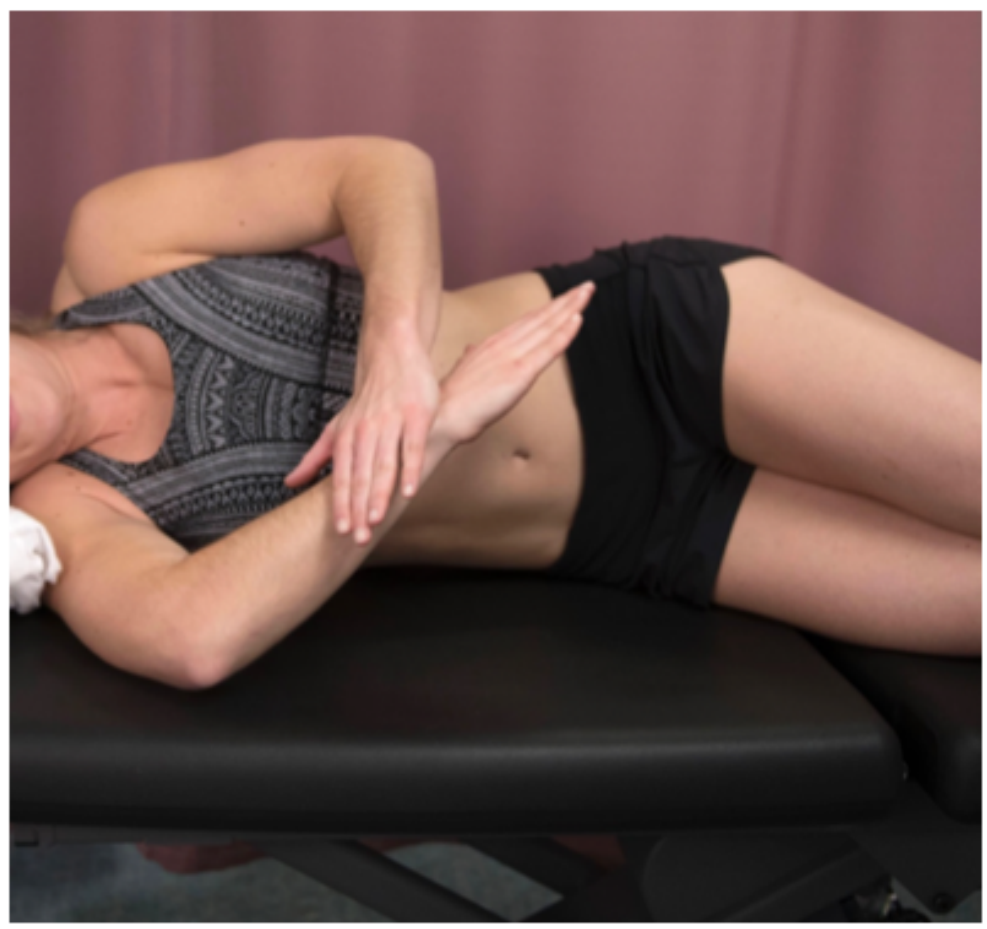

Figure 2

Modified Sleeper Stretch: Passive Glenohumeral Joint Internal Rotation. 


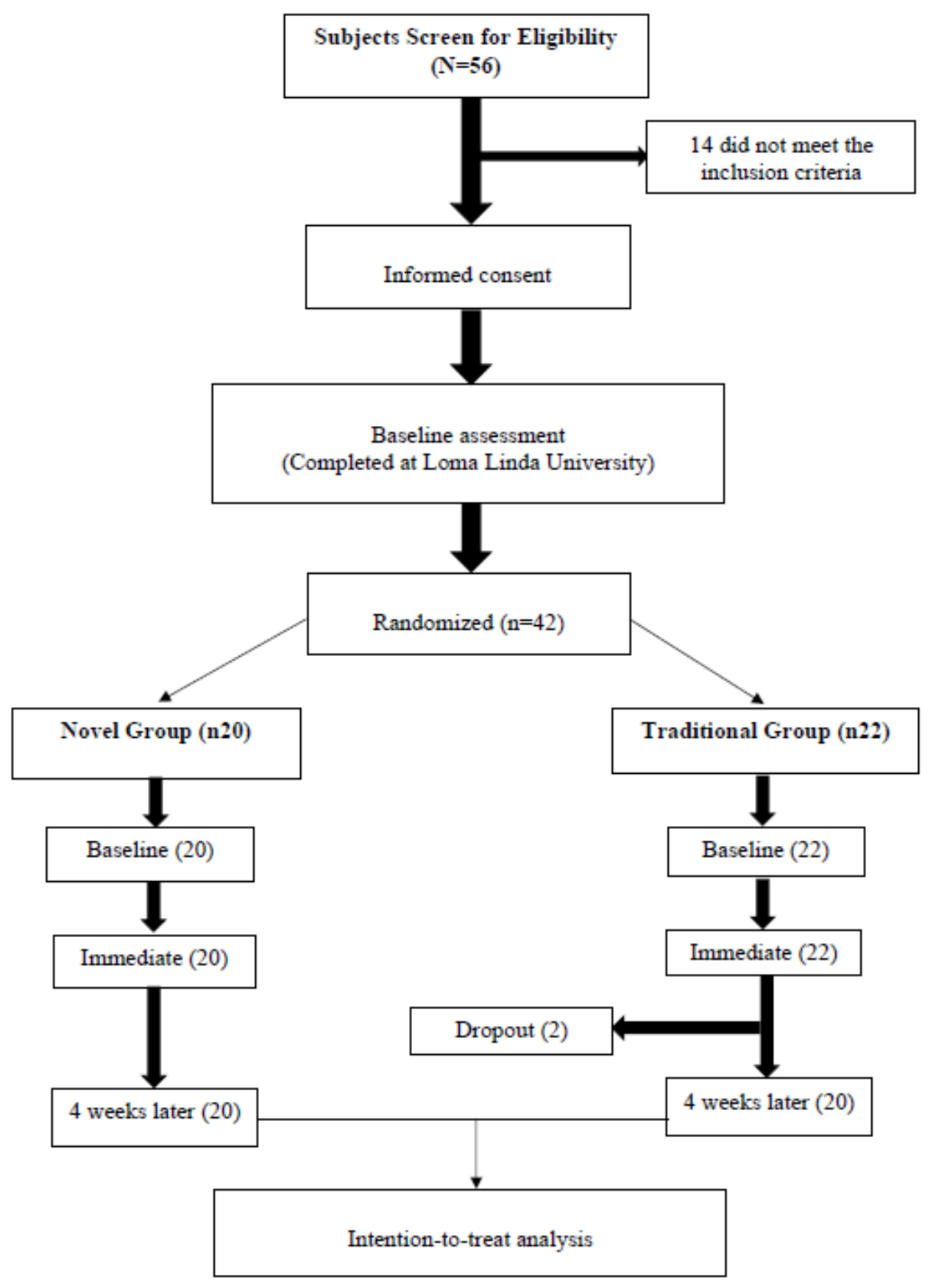

Figure 3

Flow diagram of participants' recruitment and retention.

\section{Supplementary Files}

This is a list of supplementary files associated with this preprint. Click to download.

- CONSORTChecklist.doc 